Case Report

\title{
Combined Ruxolitinib and Venetoclax Treatment in a Patient with a BCR-JAK2 Rearranged Myeloid Neoplasm
}

\author{
Coen J. Lap $\mathbb{D}^{1},{ }^{1}$ Samah Nassereddine, ${ }^{1}$ Min-Ling Liu, ${ }^{2}$ Victor E. Nava, ${ }^{2}$ and Anita Aggarwal ${ }^{1,2}$ \\ ${ }^{1}$ The George Washington University Medical Faculty Associates, Washington, DC, USA \\ ${ }^{2}$ Washington DC Veterans Affairs Medical Center, Washington, DC, USA \\ Correspondence should be addressed to Coen J. Lap; clap@mfa.gwu.edu
}

Received 22 May 2021; Revised 12 July 2021; Accepted 17 July 2021; Published 28 July 2021

Academic Editor: Håkon Reikvam

Copyright (c) 2021 Coen J. Lap et al. This is an open access article distributed under the Creative Commons Attribution License, which permits unrestricted use, distribution, and reproduction in any medium, provided the original work is properly cited.

Hematological malignancies with a BCR-JAK2 rearrangement have been described only sporadically in the literature over the last three decades. Although most patients suffer from a chronic myeloid neoplasm with marked eosinophilia, the clinical presentation varies significantly and can even manifest as a lymphoid malignancy. In this case report, we present a patient with a therapyrelated $B C R-J A K 2^{+}$myeloid neoplasm with extensive extramedullary disease localizing in the lymph nodes. While treatment with a JAK2 inhibitor (ruxolitinib) was not able to stop disease progression, combination treatment with inhibitors of both JAK2 and BCL2 (venetoclax) resulted in disease control for over 1.5 years. Combining these two inhibitors might be strategic in these patients, not only because BCL2 is a downstream target of JAK/STAT signaling but also because BCL2 is crucial for JAK2 inhibitor resistance. The recent inclusion of JAK2-rearranged malignancies in major classification systems and guidelines emphasizes the importance of not only getting a better understanding of the clinical phenotype of these rare disorders but also of identifying alternative treatment options for patients ineligible for allogeneic stem cell transplantation. Considering the low toxicity of combination treatment with these two small molecule inhibitors, this regimen could be further explored in future studies.

\section{Introduction}

Activating mutations in Janus kinase 2 (JAK2), which include $J A K 2 \mathrm{~V} 617 \mathrm{~F}$ and mutations in exon 12, are found in a high proportion of patients diagnosed with chronic myeloproliferative neoplasms (MPN), including $>95 \%$ of patients with polycythemia vera and approximately $55-65 \%$ of patients with essential thrombocytosis and primary myelofibrosis [1]. While these mutations are considered crucial molecular events in the pathogenesis of these disorders, rare chromosomal translocations involving JAK2 have been described in patients suffering from both myeloid and lymphoid malignancies $[2,3]$. The $t(9 ; 22)(\mathrm{p} 24.1 ; \mathrm{q} 11.2)$ is a recurrent rearrangement that juxtaposes the Breakpoint Cluster Region gene $(B C R ; 22 \mathrm{q} 11.2)$ to the $J A K 2$ gene $(9 \mathrm{p} 24.1)$, which leads to the formation of a $B C R-J A K 2$ fusion [4]. While this translocation has been identified in patients diagnosed with acute myeloid leukemia (AML) and B-cell acute lymphoblastic leukemia (B-ALL), most case reports have described it as the sole genetic abnormality observed in patients suffering from a clinical disease that resembles myeloid neoplasms, including MPN, myelodysplastic syndrome (MDS), or MPN/MDS overlap syndromes and is often associated with significant extramedullary hematopoiesis and/or bone marrow failure $[2,3,5-8]$. In most patients, the disease is highly therapyresistant with rapid progression occurring within the first couple of months after diagnosis [9]. While JAK2 inhibitors have been suggested to be of some benefit in controlling disease, so far, long-term remissions have only been achieved after allogeneic stem cell transplantation (allo-SCT) $[10,11]$. Here, we describe a case of a patient suffering from a therapyrelated $B C R-J A K 2^{+}$myeloid neoplasm with significant disease localized in his lymph nodes. While monotherapy with a JAK2 inhibitor (ruxolitinib) was not able to control disease, combined treatment with inhibitors of JAK2 and BCL2 (venetoclax) resulted in disease control for over 1.5 years. A rationale for combining these two small molecule inhibitors is provided as an additional option, which could be further explored for patients with this rare hematological malignancy, especially when allo-SCT is not feasible. 


\section{Case Presentation}

A 54-year-old male presented initially in February 2013 with left axillary lymphadenopathy. He was diagnosed with stage IIA nodular lymphocyte predominant Hodgkin lymphoma (NLPHL) and received two cycles of ABVD (adriamycin, bleomycin, vinblastine, and dacarbazine) before he became lost to follow-up. In March 2014, he presented again with biopsy-proven relapsing NLPHL. A bone marrow aspirate was performed at that time, revealing an unremarkable flow analysis and a normal karyotype (46XY [12]). He received three cycles of R-EPOCH (rituximab, etoposide, prednisone, vincristine, cyclophosphamide, and doxorubicin) with additional radiation therapy to the left axillary region for residual disease. He remained in complete remission until May 2018, when he presented with fatigue, a $25 \mathrm{~kg}$ weight loss over three months and was found to be anemic with a hemoglobin $(\mathrm{Hgb})$ of $7.2 \mathrm{~g} / \mathrm{dL}$, platelet count $(\mathrm{Plt})$ of $159 \times 10^{3} / \mu \mathrm{L}$, and a white blood cell count (WBC) of $6.1 \times 10^{3 /} \mu \mathrm{L}$. Peripheral blood smear revealed a neutrophilic left-shift with 3\% myelocytes, $2 \%$ metamyelocytes, and $6 \%$ eosinophils. Evaluation of the red blood cells (RBC) revealed marked anisopoikilocytosis, teardrop cells, and $2 \%$ nucleated RBC. No circulating blasts were seen. FDG-PET/CT displayed extensive FDG-avid lymphadenopathy $\left(\mathrm{SUV}_{\max } \sim 10\right)$ with splenomegaly measuring $18.4 \mathrm{~cm}$. Bone marrow (BM) biopsy demonstrated a hypercellular BM with left-shifted myeloid hyperplasia but less than $1 \%$ blasts (Figure $1(\mathrm{a})$ ), an increased myeloid to erythroid ratio $(>5: 1)$ and diffuse grade 3 reticulin fibrosis (Figure 1(b)). Cytogenetics revealed a $46 \mathrm{XY}, \operatorname{del}(6)(\mathrm{p} 21.2 \mathrm{p} 24), \mathrm{t}(22 ; 9 ; 11)$ (q11.2, p24, p11.2) [11]/46XY, del(9) [9] karyotype with fluorescent in situ hybridization (FISH) analysis showing absence of a $B C R-A B L 1$ rearrangement, but three copies of $B C R$ (Figure 2). The third copy of $B C R$ was identified to be on the short arm terminus of chromosome 9 resulting in a $B C R-J A K 2$ fusion as suggested with an additional JAK2 (9p24.1) probe (not shown). The rearrangement was confirmed with RNA sequencing, which revealed a break in exon 1 of $B C R$ (nucleotide 1107 and codon 369) and a break in exon 17 of $J A K 2$ (nucleotide 2171 and codon 724 ) leading to an in-frame fusion. The resulting product included the coiled-coil oligodimerization domain of $B C R$ as well as the area distal to the pseudokinase domain (JH2) of $J A K 2$, thereby maintaining the active tyrosine kinase domain (JH1) in exon 19. Additional mutation analysis was negative for $J A K 2$ V617 F or alterations in CALR, PDGFRA, PDGFRB, or FGFR1 but did reveal an alteration in CD36 (c.157_158AA > G_p.N53 fs*24). Excisional biopsy of a lymph node ruled out lymphoma, but revealed extensive extramedullary involvement of the same myeloid malignancy with prominent erythroid precursors, atypical megakaryocytes, and left-shifted myeloid cells without increase of blasts (Figure 3). Importantly, the same BCR-JAK2 translocation was identified in the biopsy and confirmed with RNA sequencing.

Pending the results of the molecular analysis, the patient was started on the $B C R-A B L 1$ tyrosine kinase inhibitor (TKI) imatinib mesylate $400 \mathrm{mg}$ once daily due to an initial concern of CML. Because of limited response and upon identification of the $B C R-J A K 2$ translocation, treatment was switched to ruxolitinib and uptitrated to $20 \mathrm{mg}$ twice daily. An early referral for allo-SCT was discussed, but the patient decided to postpone this. While initially an improvement in blood counts was observed ( $\mathrm{Hgb}, 10.8 \mathrm{~g} / \mathrm{dL}$; Plt, $180 \times 10^{3}$ / $\mu \mathrm{L}$; WBC, $8.1 \times 10^{3 /} \mu \mathrm{L}$ ) with a reduced need for transfusions, he developed worsening anemia $(\mathrm{Hgb}, 7.8 \mathrm{~g} / \mathrm{dL})$, thrombocytopenia (Plt, $30 \times 10^{3} / \mu \mathrm{L}$ ), and leukocytosis (WBC, $\left.17.6 \times 10^{3 /} \mu \mathrm{L}\right)$ with an elevated $\mathrm{LDH}(680 \mathrm{U} / \mathrm{L})$ in January 2019. A peripheral smear revealed $6 \%$ promyelocytes, $3 \%$ metamyelocytes, $6 \%$ eosinophils, and $2 \%$ blasts, but repeated bone marrow evaluation only showed diffuse fibrosis and the previously identified $B C R-J A K 2$ translocation. The spleen remained enlarged at $19 \mathrm{~cm}$ on imaging. Ruxolitinib was continued, and because of disease progression, venetoclax was initiated and uptitrated to $400 \mathrm{mg}$ daily. At that point, he was evaluated for allo-SCT but was found ineligible due to diminished pulmonary function. After several weeks, blood counts improved, and the patient became transfusion-independent and EPO support was discontinued. Over time, his blood counts normalized (Hgb, $12.4 \mathrm{~g} / \mathrm{dl}$; Plt, $167 \times 10^{3} /$ $\mu \mathrm{L}$; WBC, $5.7 \times 10^{3} / \mu \mathrm{L}$ with $2 \%$ eosinophils) with repeat imaging, six months after the start of venetoclax, revealing a significant decrease in lymphadenopathy and a reduction in the spleen size to $13.1 \mathrm{~cm}$. A new BM biopsy showed stable disease with extensive fibrosis, no increase in blasts, but persistence of the BCR-JAK2 fusion and the CD36 alteration, as well as a new TET2 mutation (c.3782 G > A_p.R1261H). During this time, the patient tolerated the combination regimen well without development of infections, need for blood product support, or hospitalizations. Although no cytogenetic response was observed, the treatment significantly improved his quality of life. He remained stable under this combination treatment until May 2020, at which point he presented with abdominal complaints and renal failure as a result of bulky lymphadenopathy and splenomegaly due to disease progression. Ruxolitinib and venetoclax were discontinued. Blood counts revealed $\mathrm{Hgb}, 11 \mathrm{~g} / \mathrm{dL} ; \mathrm{Plt}$, $170 \times 10^{3} / \mu \mathrm{L}$; and $\mathrm{WBC}, 12.1 \times 10^{3} / \mu \mathrm{L}$ with a peripheral smear showing left-shifted hematopoiesis with $4 \%$ eosinophils and $1 \%$ blasts. Renewed bone marrow analysis only revealed fibrosis without a significant increase in blasts. The patient was started on azacytidine and re-evaluated for alloSCT. However, allo-SCT was further postponed as a result of the patient contracting COVID-19. During that period, azacytidine was continued, and ruxolitinib was restarted. Unfortunately, the disease progressed with worsening bone marrow failure, without signs of transformation, and he passed away several months later.

\section{Discussion}

The 2017 revision of the World Health Organization (WHO) Classification of Myeloid Neoplasms and Acute Leukemia included a new provisional entity "Myeloid Neoplasm with PCM1-JAK2 Rearrangement" in the category of "Myeloid/Lymphoid Neoplasms Associated with Eosinophilia and Rearrangement of PDGFRA, PDGFRB, or FGFR1, 


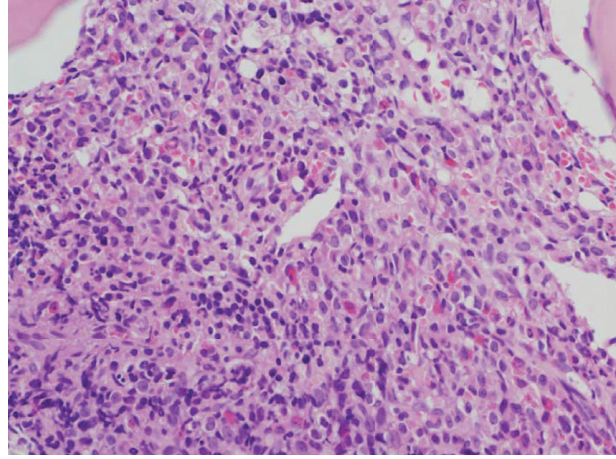

(a)

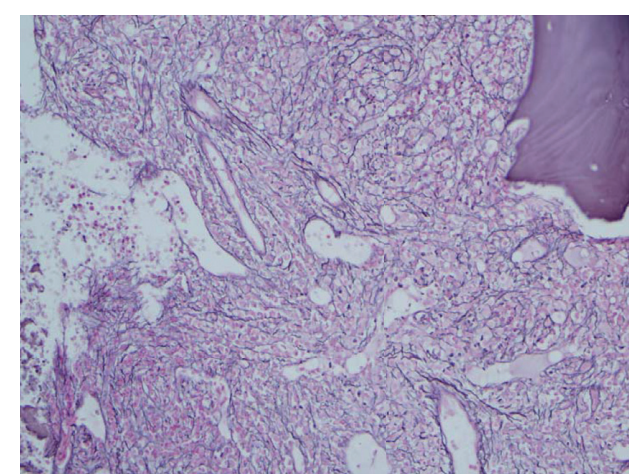

(b)

FIGURE 1: Bone marrow biopsy showing a hypercellular marrow with marked myeloid hyperplasia with left shift ((a) hematoxylin and eosin stain, 400x) and grade 3 fibrosis ((b) reticulin stain, 200x).

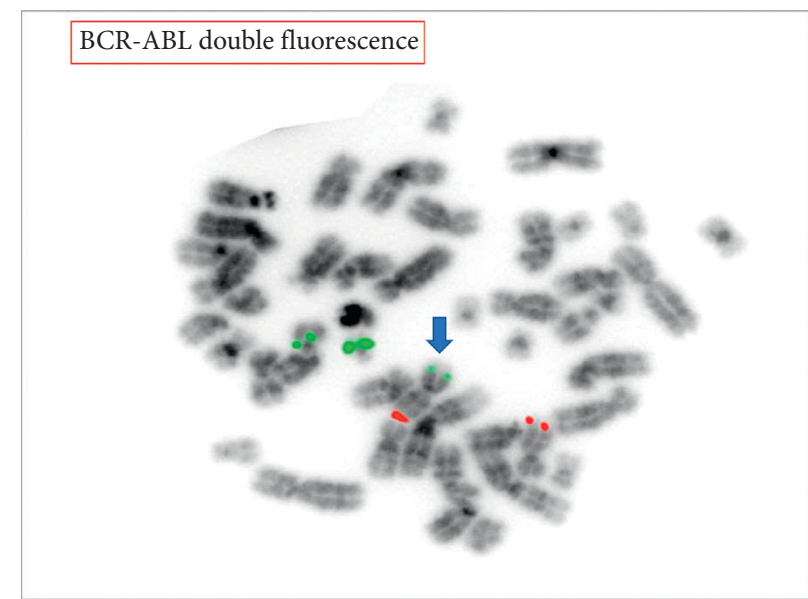

Figure 2: Fluorescent in situ hybridization with probes for $B C R$ (22q11.2, green signal) and $A B L 1$ (9q34.1, red signal) was negative for a $B C R-A B L 1$ rearrangement. However, a third signal for the $B C R$ probe (green signal, arrow) was observed, suggesting partial or complete gain of chromosome 22 or a translocation that involves $B C R$ with a partner gene other than $A B L 1$. Additional testing with a probe for JAK2 (9p24.1, not shown) suggested the presence of a BCR-JAK2 fusion, which was confirmed with RNA sequencing.

or with PCM1-JAK2" [12]. While the patient described here does not have a hematological malignancy with a PCM1$J A K 2$ rearrangement, many experts in the field advocate to classify those rare cases with a JAK2 rearrangement, including gene partners other than PCM1, in the same category because of similar clinical features [13]. This is also highlighted by the inclusion of patients with a BCR-JAK2 rearrangement in the latest version of the U.S. National Comprehensive Cancer Network (NCCN) guidelines for "Myeloid/Lymphoid Neoplasms with Eosinophilia and Tyrosine Kinase Fusion Genes" (version 3.2021). Regardless of the classification, the presence of a BCR-JAK2 translocation has not been linked to a specific clinical phenotype although most patients present as a chronic myeloid neoplasm with marked eosinophilia ( $50-70 \%)$ (Table 1$)$. However, eosinophilia can be notably absent and is not a requirement for the diagnosis. Additionally, BCR-JAK2 rearrangements are sometimes identified in the new provisional entity of Philadelphia chromosome-like ALL, which are characterized by activated tyrosine kinase signaling as well as JAK/STAT signaling but lack the presence of a $B C R-A B L 1$ rearrangement [19]. The clinical heterogeneity may be the result of both disease factors (additional mutations and subclones) as well as existing host factors (germline allele diversity). Our patient presented with symptoms of a myeloid malignancy with a newly acquired $B C R-J A K 2$ rearrangement, four years after treatment for relapsing NLPHL. Considering that the patient had a normal bone marrow evaluation at the time of his relapsing NLHPL and was subsequently exposed to cytotoxic chemotherapy, including etoposide and cyclophosphamide, it supports a diagnosis of a therapy-related malignancy. It is likely that clonal evolution and expansion over time have resulted in a secondary myeloid neoplasm. Although the clinical significance of a CD36 N53 fs*24 mutation is not clear at the moment, it is important to mention that it has been identified in patients with mixed phenotype acute leukemia's (MPAL) [20]. More importantly, the R1261H missense mutation in the epigenetic modifier TET2 is a recurrent abnormality identified in (therapy-related) myeloid malignancies and is considered a driver of clonal hematopoiesis [21]. 


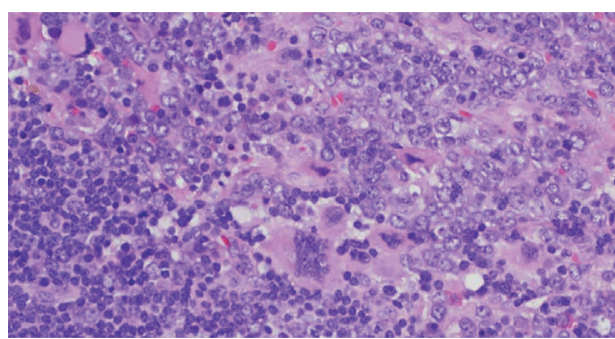

(a)

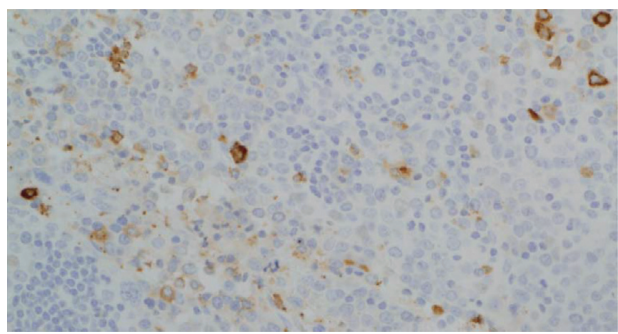

(c)

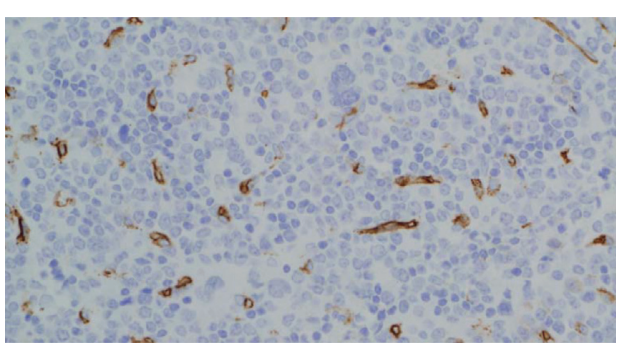

(b)

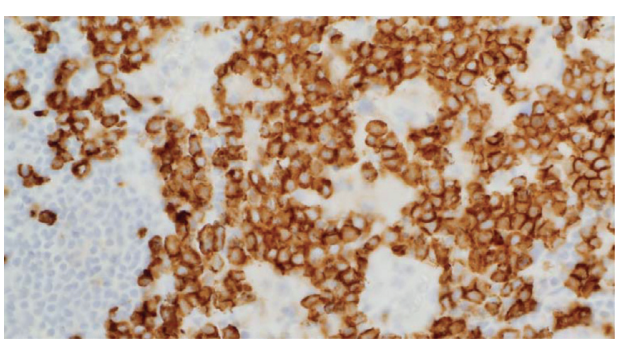

(d)

Figure 3: Lymph node showing extensive extramedullary involvement by a myeloproliferative neoplasm with numerous megakaryocytes ((a) hematoxylin and eosin stain, 400x), no increase in blasts ((b) CD34 immunostain, 400x), or significant myeloid cells ((c) MPO immunostain, 400x) but with prominent erythroid precursors ((d) CD71 immunostain, 400x).

TABLE 1: Summary of the literature regarding described phenotype, treatment, and response duration of patients with a BCR-JAK2 fusion.

\begin{tabular}{|c|c|c|c|c|c|}
\hline Reference & Age & Sex & Disease & Treatment & Follow-up \\
\hline Griesinger et al. [2] & 63 & $\mathrm{~F}$ & $\begin{array}{l}\text { CML-like MPD, } \\
\text { myeloid blast crisis }\end{array}$ & $\begin{array}{l}\text { Imatinib, hydroxyurea, } \\
\text { interferon-alpha }\end{array}$ & $\begin{array}{l}\text { Complete hematological response. } 20 \text { months after } \\
\text { diagnosis, she developed myeloid blast crisis and died. }\end{array}$ \\
\hline $\begin{array}{l}\text { Kantarcioglu et al. } \\
\text { [3] }\end{array}$ & 64 & $\mathrm{~F}$ & MDS & Not described & Died 3 months after diagnosis \\
\hline $\begin{array}{l}\text { Cuesta- } \\
\text { Domínguez et al. } \\
{[4]}\end{array}$ & 58 & M & B-ALL & Not described & Not described \\
\hline Cirmena et al. [5] & 67 & $\mathrm{~F}$ & AML & Not described & Not described \\
\hline Tirado et al. [6] & 14 & M & B-ALL & Not described & Not described \\
\hline Snider et al. [7] & 59 & M & $\begin{array}{l}\text { MPN with } \\
\text { eosinophilia, MPAL }\end{array}$ & $\begin{array}{l}\text { Hydroxyurea, } \\
\text { chemotherapy, allo- } \\
\text { SCT }\end{array}$ & $\begin{array}{c}\text { Transformation to MPAL } 12 \text { months after diagnosis. No } \\
\text { evidence of disease } 30 \text { days after allo-SCT. }\end{array}$ \\
\hline Elnaggar et al. [8] & 84 & M & CML-like MPD & Imatinib & Lost to follow-up \\
\hline Schwaab et al. [10] & $\mathrm{n} / \mathrm{a}$ & $\mathrm{n} / \mathrm{a}$ & Myeloid neoplasm & $\begin{array}{l}\text { Ruxolitinib } 20 \mathrm{mg} \text { BID, } \\
\text { allo-SCT }\end{array}$ & $\begin{array}{l}\text { Complete hematological and cytogenetic remission on } \\
\text { ruxolitinib. Relapsed after } 18 \text { months upon which } \\
\text { referred for allo-SCT. }\end{array}$ \\
\hline Schwaab et al. [11] & 69 & M & MDS/MPN & $\begin{array}{l}\text { Ruxolitinib } 20 \mathrm{mg} \text { BID, } \\
\text { allo-SCT }\end{array}$ & $\begin{array}{l}\text { Complete hematological, cytogenetic, and molecular } \\
\text { response. AlloSCT while in remission }\end{array}$ \\
\hline He et al. [14] & 36 & $\mathrm{~F}$ & $\begin{array}{l}\text { MPN with } \\
\text { eosinophilia }\end{array}$ & Dasatinib, allo-SCT & $\begin{array}{c}\text { No response of dasatinib. No evidence of disease } 18 \\
\text { months after allo-SCT. }\end{array}$ \\
\hline Bellesso et al. [15] & 54 & M & $B C R-A B L^{-} \mathrm{CML}$ & $\begin{array}{l}\text { Imatinib, dasatinib, } \\
\text { allo-SCT }\end{array}$ & $\begin{array}{c}\text { No response of imatinib or dasatinib. Died of aGvHD } 53 \\
\text { days after allo-SCT }\end{array}$ \\
\hline Impera et al. [16] & 49 & $\mathrm{~F}$ & MPN-unclassifiable & $\begin{array}{l}\text { Imatinib, dasatinib peg- } \\
\text { interferon }\end{array}$ & $\begin{array}{l}\text { No initial response with imatinib or dasatinib. Partial } \\
\text { hematological response with peg-interferon }\end{array}$ \\
\hline Lane et al. [17] & 44 & M & $\begin{array}{l}\text { Atypical CML with } \\
\text { leukemia cutis }\end{array}$ & Not described & Not described \\
\hline Thakral et al. [18] & 31 & M & $\begin{array}{l}\text { MPN with } \\
\text { eosinophilia }\end{array}$ & Hydroxyurea, allo-SCT & No evidence of disease three months posttransplantation \\
\hline
\end{tabular}

CML, chronic myeloid leukemia; MPD, myeloproliferative disorder; MDS, myelodysplastic syndrome; B-ALL, B-cell acute lymphoblastic leukemia; AML, acute myeloid leukemia; MPN, myeloproliferative neoplasm; MPAL, mixed phenotype acute leukemia; allo-SCT, allogeneic stem cell transplantation; BID, twice a day; aGvHD, acute graft-versus-host disease; F, female; M, male. 
As a result of the $t(9 ; 22)(\mathrm{p} 24.1 ; \mathrm{q} 11.2)$, a fusion gene is formed in which the oligodimerization domain of $B C R$ is juxtaposed to the JAK2 tyrosine kinase domain resulting in constitutive activation of the JAK/STAT signaling pathway, which promotes cellular proliferation, differentiation, and growth $[4,22]$. JAK inhibitors such as ruxolitinib (JAK1/2) have been used successfully in patients with JAK2-mutated MPN and, as such, have been proposed as a possible treatment for patients with a $B C R-J A K 2^{+}$myeloid malignancy $[10,11]$. Unfortunately, responses seen in the handful of patients treated to date have been mixed with some progressing rapidly and others obtaining a complete cytogenetic response. However, even cytogenetic responses are usually short-lived due to the development of JAK2 inhibitor resistance. Although our patient had an initial response after the start of ruxolitinib, he progressed five months later, prompting initiation of the BCL2 inhibitor venetoclax. Combining these two inhibitors may be strategic since downstream transcriptional targets of JAK/ STAT signaling include antiapoptotic members of the BCL2 family, such as BCL2 and BCL-xl [23-25]. Several preclinical studies have suggested a beneficial synergistic effect of combining these two inhibitors in patients with JAK2-mutated hematological malignancies [25-27]. Moreover, resistance to JAK2 inhibitors has been attributed to overexpression of both BCL2 and BCL-xl. Although our patient did not develop a cytogenetic remission, the combination treatment stabilized the disease for over 1.5 years, achieving normal blood counts and significant reduction in splenomegaly and lymphadenopathy before final progression.

Even with combination treatment, our patient eventually developed progressive disease and succumbed before he was able to undergo allo-SCT. Although combined treatment might be able to control disease for a significant period of time, even when monotherapy with a JAK2 inhibitor fails, only allo-SCT results in long-term disease control, affirming the need for early referral. It needs to be emphasized that TKIs used for the treatment of $B C R-A B L 1^{+}$CML have not shown to be of any benefit and should be avoided in the treatment of these patients [11]. Considering the rarity of hematological malignancies with a $B C R-J A K 2$ translocation, it remains important to report cases to disseminate clinical experience, which may allow better characterization of the clinical phenotype and provide beneficial insights regarding targeted therapy regimens for patients who are not eligible for allo-SCT.

\section{Conclusion}

$B C R-J A K 2$-rearranged hematological malignancies are rare disorders that can have a variable clinical presentation but should be classified as a "Myeloid/Lymphoid Neoplasms with Eosinophilia and Gene Rearrangement." Because eosinophilia can be discrete, or even absent, it highlights the importance of cytogenetic analysis in all patients that are presenting with a myeloid neoplasm. Combination treatment with inhibitors of JAK2 and BCL2 has minimal toxicity and can be beneficial in controlling disease.

\section{Data Availability}

The data used to support the findings of this study are available from the corresponding author upon request.

\section{Consent}

Written informed consent was obtained from the patient for publication of this case report and any accompanying images.

\section{Conflicts of Interest}

The authors declare that they have no conflicts of interest.

\section{Authors' Contributions}

All authors contributed equally to this work.

\section{References}

[1] A. Tefferi, "Novel mutations and their functional and clinical relevance in myeloproliferative neoplasms: JAK2, MPL, TET2, ASXL1, CBL, IDH and IKZF1," Leukemia, vol. 24, no. 6, pp. 1128-1138, 2010.

[2] F. Griesinger, H. Hennig, F. Hillmer et al., "ABCR-JAK2 fusion gene as the result of a $\mathrm{t}(9 ; 22)(\mathrm{p} 24 ; \mathrm{q} 11.2)$ translocation in a patient with a clinically typical chronic myeloid leukemia," Genes, Chromosomes and Cancer, vol. 44, no. 3, pp. 329-333, 2005.

[3] B. Kantarcioglu, I. Kaygusuz-Atagunduz, A. Uzay, T. Toptas, T. F. Tuglular, and M. Bayik, "Myelodysplastic syndrome with $\mathrm{t}(9 ; 22)(\mathrm{p} 24 ; \mathrm{q} 11.2)$, a BCR-JAK2 fusion: case report and review of the literature," International Journal of Hematology, vol. 102, no. 3, pp. 383-387, 2015.

[4] Á. Cuesta-Domínguez, M. Ortega, C. Ormazábal et al., "Transforming and tumorigenic activity of JAK2 by fusion to BCR: molecular mechanisms of action of a novel BCR-JAK2 tyrosine-kinase," PLoS One, vol. 7, no. 2, Article ID e32451, 2012.

[5] G. Cirmena, S. Aliano, G. Fugazza et al., "A BCR-JAK2 fusion gene as the result of a $\mathrm{t}(9 ; 22)(\mathrm{p} 24 ; \mathrm{q} 11)$ in a patient with acute myeloid leukemia," Cancer Genetics and Cytogenetics, vol. 183 , no. 2, pp. 105-108, 2008.

[6] C. A. Tirado, W. Chen, L. J.-s. Huang et al., "Novel JAK2 rearrangement resulting from a $\mathrm{t}(9 ; 22)(\mathrm{p} 24 ; \mathrm{q} 11.2)$ in B-acute lymphoblastic leukemia," Leukemia Research, vol. 34, no. 12, pp. 1674-1676, 2010.

[7] J. S. Snider, I. Znoyko, K. G. Lindsey et al., "Integrated genomic analysis using chromosomal microarray, fluorescence in situ hybridization and mate pair analyses: characterization of a cryptic $\mathrm{t}(9 ; 22)$ (p24.1;q11.2)/BCR-JAK2 in myeloid/lymphoid neoplasm with eosinophilia," Cancer Genetics, vol. 247, pp. 44-47, 2020.

[8] M. M. Elnaggar, S. Agersborg, T. Sahoo et al., "BCR-JAK2 fusion as a result of a translocation $(9 ; 22)(\mathrm{p} 24 ; \mathrm{q} 11.2)$ in a patient with CML-like myeloproliferative disease," Molecular Cytogenetics, vol. 5, no. 1, p. 23, 2012.

[9] C. A. Smith and G. Fan, "The saga of JAK2 mutations and translocations in hematologic disorders: pathogenesis, diagnostic and therapeutic prospects, and revised World Health Organization diagnostic criteria for myeloproliferative neoplasms," Human Pathology, vol. 39, no. 6, pp. 795-810, 2008. 
[10] J. Schwaab, M. Knut, C. Haferlach et al., "Limited duration of complete remission on ruxolitinib in myeloid neoplasms with PCM1-JAK2 and BCR-JAK2 fusion genes," Annals of Hematology, vol. 94, no. 2, pp. 233-238, 2015.

[11] J. Schwaab, N. Naumann, J. Luebke et al., "Response to tyrosine kinase inhibitors in myeloid neoplasms associated with PCM1 - JAK2 , BCR-JAK2 and ETV6-ABL1 fusion genes," American Journal of Hematology, vol. 95, no. 7, pp. 824-833, 2020.

[12] S. Swerdlow, E. Campo, N Harris et al., WHO Classification of Tumours of Haematopoietic and Lymphoid Tissues, iARC Press, Lyon, France, 4th edition, 2017.

[13] A. Reiter and J. Gotlib, "Myeloid neoplasms with eosinophilia," Blood, vol. 129, no. 6, pp. 704-714, 2017.

[14] R. He, P. T. Greipp, A. Rangan et al., "BCR-JAK2 fusion in a myeloproliferative neoplasm with associated eosinophilia," Cancer Genetics, vol. 209, no. 5, pp. 223-228, 2016.

[15] M. Bellesso, R. Santucci, D. F. Dias, R. Centrone, and R. C. Elias, "Atypical chronic myeloid leukemia with $\mathrm{t}(9$; 22)(p24, 11.2), a BCR-JAK2 fusion gene," Revista Brasileira de Hematologia e Hemoterapia, vol. 35, pp. 218-219, 2013.

[16] L. Impera, A. Lonoce, D. A. Fanfulla et al., "Two alternatively spliced $5^{\prime} \mathrm{BCR} / 3^{\prime} \mathrm{JAK} 2$ fusion transcripts in a myeloproliferative neoplasm with a three-way $\mathrm{t}(9 ; 18 ; 22)(\mathrm{p} 23 ; \mathrm{p} 11.3 ; \mathrm{q} 11.2)$ translocation," Cancer Genetics, vol. 204, no. 9, pp. 512-515, 2011.

[17] S. W. Lane, D. J. Fairbairn, C. McCarthy, A. Nandini, J. PerryKeene, and G. A. Kennedy, "Leukaemia cutis in atypical chronic myeloid leukaemia with a $\mathrm{t}(9 ; 22)$ (p24; q11.2) leading to BCR-JAK2 fusion," British Journal of Haematology, vol. 142 , no. 4 , p. $503,2008$.

[18] B. Thakral, T. Muzzafar, S. A. Wang, and L. J. Medeiros, "Myeloid neoplasm with eosinophilia and BCR-JAK2/t $(9$; 22)(p24; q11.2) morphologically mimicking chronic myeloid leukemia," Annals of Diagnostic Pathology, vol. 44, Article ID $151405,2020$.

[19] S. Jain and A. Abraham, "BCR-ABL1-like B-acute lymphoblastic leukemia/lymphoma: a comprehensive review," $A r$ chives of Pathology \& Laboratory Medicine, vol. 144, no. 2, pp. 150-155, 2020.

[20] B. M. Getta, M. Roshal, J. Zheng et al., "Allogeneic hematopoietic stem cell transplantation with myeloablative conditioning is associated with favorable outcomes in mixed phenotype acute leukemia," Biology of Blood and Marrow Transplantation, vol. 23, no. 11, pp. 1879-1886, 2017.

[21] R. L. Bowman, L. Busque, and R. L. Levine, "Clonal hematopoiesis and evolution to hematopoietic malignancies," Cell Stem Cell, vol. 22, no. 2, pp. 157-170, 2018.

[22] E. Bousoik and H. Montazeri Aliabadi, "Do we know Jack" about JAK? A closer look at JAK/STAT signaling pathway," Frontiers in Oncology, vol. 8, p. 287, 2018.

[23] P. Sepúlveda, A. Encabo, F. Carbonell-Uberos, and M. D. Miñana, "BCL-2 expression is mainly regulated by JAK/ STAT3 pathway in human CD34+ hematopoietic cells," Cell Death \& Differentiation, vol. 14, no. 2, pp. 378-380, 2007.

[24] I. Sakai and A. S. Kraft, "The kinase domain of Jak2 mediates induction of bcl-2 and delays cell death in hematopoietic cells," Journal of Biological Chemistry, vol. 272, no. 19, pp. 12350-12358, 1997.

[25] A. Zeuner, F. Pedini, F. Francescangeli et al., "Activity of the BH3 mimetic ABT-737 on polycythemia vera erythroid precursor cells," Blood, vol. 113, no. 7, pp. 1522-1525, 2009.

[26] B. Will, T. Siddiqi, M. A. Jordà et al., "Apoptosis induced by JAK2 inhibition is mediated by Bim and enhanced by the $\mathrm{BH} 3$ mimetic ABT-737 in JAK2 mutant human erythroid cells," Blood, vol. 115, no. 14, pp. 2901-2909, 2010.

[27] M. Waibel, V. S. Solomon, D. A. Knight et al., "Combined targeting of JAK2 and BCL-2/BCL-XL to cure mutant JAK2driven malignancies and overcome acquired resistance to JAK2 inhibitors," Cell Reports, vol. 5, no. 4, pp. 1047-1059, 2013. 